\title{
Ectoparásitos (Argulidae, Cymothoidae, Corallanidae) en rayas de agua dulce (Potamotrygonidae) de la Orinoquia colombiana
}

Ectoparasites (Argulidae, Cymothoidae, Corallanidae) in freshwater rays (Potamotrygonidae) of the Colombian Orinoquia

\section{Carlos A. Lasso, Martha R. Campos, Mónica A. Morales-Betancourt y David Castro}

\section{Resumen}

Se registra por primera vez para Colombia (cuenca del Orinoco), cuatro especies de crustáceos ectoparásitos en rayas de agua dulce (Potamotrygonidae), género Potamotrygon. Dos que pertenecen a la subclase Branchiura, familia Argulidae: Dolops reperta (Bouvier, 1899) y D. striata (Bouvier, 1899) y dos al orden Isopoda, correspondientes a las familias Cymothoidae y Corallanidae, representadas con las especies Braga cigarra (Szidat \& Schubart, 1960) y Excorallana delaneyi (Stone \& Heard, 1989), respectivamente.

Palabras clave. Branchiura. Isopoda. Peces. Potamotrygon. Cuenca del río Orinoco.

\begin{abstract}
Four species of ectoparasite crustaceans are registered for the first time from the Orinoco River Basin (Colombia) in freshwater stingrays of the family Potamotrygonidae, genus Potamotrygon. Two belong to the Branchiura subclass, Argulidae family: Dolops reperta (Bouvier, 1899) and D. striata (Bouvier, 1899), and two to the Isopoda Order, corresponding to the families Cymothoidae and Corallanidae, represented by the species Braga cigarra (Szidat \& Schubart, 1960) and Excorallana delaneyi (Stone \& Heard, 1989), respectively.
\end{abstract}

Keywords. Branchiura. Fishes. Isopoda. Orinoco River Basin. Potamotrygon.

\section{Introducción}

Los estudios relativos a la parasitosis de rayas de agua dulce en Colombia son escasos y solo existen algunos registros sobre las especies de endoparásitos descritos o citados para el país y sus respectivos hospedadores, como Potamotrygon magdalenae (Brooks y Thorson, 1976; Brooks et al., 1979, 1981), Potamotrygon constellata (Deardoff et al., 1981), Potamotrygon orbignyi, Potamotrygon scobina y Paratrygon aiereba, estas tres últimas especies parasitadas por peces hematófagos (Vandelliinae) (Lasso et al., 2015). A nivel continental hay mayor información sobre endoparásitos que ectoparásitos, y la mayoría de los estudios se relacionan con especies del 
género Argulus, infestando varias especies de rayas del género Potamotrygon en Brasil (Peralta et al., 1998; Domingues y Marques, 2010; Lemos et al., 2010; Gama et al., 2015). Hay un registro de Dolops sp., parasitando a Potamotrygon leopoldi y Paratrygon aiereba, en los ríos Xingú y Amazonas, respectivamente (Domingues y Marques, 2010). Gama (2016) en la revisión más reciente sobre parasitosis de rayas de agua dulce en Suramérica, estableció que a nivel de crustáceos ectoparásitos solo se conocen registros de Argulus juruparensis, Argulus cf. flavescens y Dolops sp. Estudios previos en la Orinoquia venezolana (Weibezahn y Cobo, 1964), han encontrado especies de Dolops, pero siempre en peces óseos, nunca en rayas. Hay registros de nueve especies de este género para la región neotropical (Thatcher, 1991).

Los isópodos ectoparásitos de peces neotropicales han sido poco estudiados. Los géneros y especies no están completamente identificadas. En Suramérica existen registros de un gran número de especies de agua dulce de la familia Cymothoidae (9 géneros y 27 especies). No se conocen registros de la familia Cymothoidae de agua dulce en Norteamérica ni Europa y solo hay cuatro especies reportadas para África (Thatcher, 2006).

En este trabajo se registra por primera vez para la Orinoquia colombiana, la ectoparasitosis de cuatro especies de crustáceos en rayas de agua dulce de la familia Potamotrygonidae, dos especies pertenecientes a la subclase Branchiura y dos al orden Isopoda.

\section{Materiales y métodos}

Se examinaron 350 ejemplares provenientes de capturas dirigidas al inventario y estudios bioecológicos, incluyendo las cuatro especies de rayas descritas para la Orinoquia: Potamotrygon motoro (Müller \& Henle, 1841), P. orbignyi (Castelnau, 1855), P. schroederi FernándezYépez, 1957, P. scobina Garman, 1913 y cuatro especies nuevas para la ciencia: dos del género Potamotrygon (Lasso y Morales-Betancourt, obs. pers.) y dos de Paratrygon (denominadas sp. 1 y sp. 2), estas últimas en proceso de descripción. Los individuos fueron colectados en el cauce principal del río Orinoco, planicie inundable y afluentes (caño Dagua, río Bita, caño Negro y RNP Bojonawi), departamento del Vichada, Orinoquia colombiana (Figura 1), durante la estación seca desde el 2013 al 2017, mediante el uso de anzuelos, redes de ahorque (trasmallos), arpones y captura manual (redes de mano).

Para la localización de los ectoparásitos se efectuó una revisión manual posterior al momento de la captura, de la superficie ventral, dorsal, bucal y cavidad branquial de los diferentes especímenes de rayas. Tras esta revisión, en Potamotrygon motoro y $P$. orbignyi fueron encontrados ectoparásitos argúlidos (un ejemplar por especie) y en P. orbignyi, un isópodo. En el caso particular de Potamotrygon schroederi, los especímenes fueron capturados y transportados en envases adecuados para ser mantenidos en cautiverio por un año en la Estación Piscícola de la Fundación Orinoquia en Puerto Carreño (FO). Al llegar a la Estación los ectoparásitos fueron extraídos con el método propuesto por Álvarez-Perdomo et al. (2016), consistente en usar permanganato de potasio $\left(\mathrm{KMnO}_{4}\right)$ a razón de $1 \mathrm{~g} / \mathrm{m}^{3}$ en el estanque durante tres días.

El material colectado fue preservado en etanol $70 \%$. Las rayas fueron depositadas en la colección de peces del Instituto de Investigación de Recursos Biológicos Alexander von Humboldt (IAvH) y los crustáceos fueron depositados en la colección de Crustáceos del Instituto de Ciencias Naturales de la Universidad Nacional de Colombia (ICN).

\section{Resultados}

De los 350 especímenes examinados, y corres-pondientes a seis especies de rayas (Paratrygon sp. 1, Paratrygon sp. 2, Potamotrygon motoro, P. orbignyi, P. scobina, P. schroederi y Potamotrygon sp.), tan solo en tres ejemplares de las especies Potamotrygon motoro (IAvH- 12425 y 16142), Potamotrygon orbignyi (IAvH-12829) y Potamotrygon schroederi, se observaron crustáceos ectoparásitos (Figura 2). 




Figura 1. Mapa del área de estudio, indicando las principales zonas de muestreo.


Figura 2. Especies de rayas con presencia de ectoparásitos. A. Potamotrygon motoro. B. Potamotrygon orbignyi. C. Potamotrygon schroederi. Foto: Mónica Morales-Betancourt. 
Clase Maxillopoda

Subclase Branchiura

Familia Argulidae Leach, 1819

Dolops reperta (Bouvier, 1899) (Figura 3)

Material examinado. Puerto Carreño, Reserva Natural Bojonawi (RNB), Laguna El Pañuelo, departamento del Vichada, $06^{\circ} 06^{\prime} 8,3^{\prime \prime} \mathrm{N}$ $74^{\circ} 02^{\prime} 21,0^{\prime \prime} \mathrm{O}, 5$ de abril de 2014; colectó C. A. Lasso y M. A. Morales-Betancourt; 1 macho, 1 espécimen fragmentado, ICN-MHN-CR 3207. Hospedero: Potamotrygon motoro, macho adulto $330 \mathrm{~mm}$ ancho discal (AD) y $1,9 \mathrm{~kg}(\mathrm{IAvH}-12425)$ y Potamotrygon schroederi (espécimen macho adulto mantenido en cautiverio en la Estación Piscícola en Puerto Carreño).

Diagnosis. Caparazón semicircular; primeras maxilas anchas en la base y terminadas en uñas; placas basales de las segundas maxilas provistas de tres dientes; lóbulos abdominales cortos, anchos y de similar longitud (Thatcher, 2006).

Comentarios. Dolops reperta se colectó en la superficie del disco, cerca de los espiráculos, en un macho adulto (330 $\mathrm{mm} \mathrm{AD)} \mathrm{de} \mathrm{Potamotrygon}$ motoro (IAvH-12425) de la laguna El PañueloRNB (planicie inundable río Orinoco). También se observó y se colectó posteriormente, moviéndose activamente en toda la superficie discal de Potamotrygon schroederi (macho adulto) colectado en el caño Dagua y mantenido en cautiverio en la Estación Piscícola de Puerto Carreño. Estos ectoparásitos llegaron con las rayas provenientes del medio natural.

Las especies del género Dolops se desplazan libremente nadando con sus apéndices torácicos y fijándose de nuevo en diferentes partes del pez (Weibezahn y Cobo, 1964).

Potamotrygon motoro es una especie de amplia distribución presente en diferentes tipos de hábitats lóticos y lénticos y tipos de agua (negras, claras y blancas), su mayor actividad es nocturna y es capaz de colonizar muchos hábitats, de acuerdo con el periodo de inundación (Lasso et al., 2016).
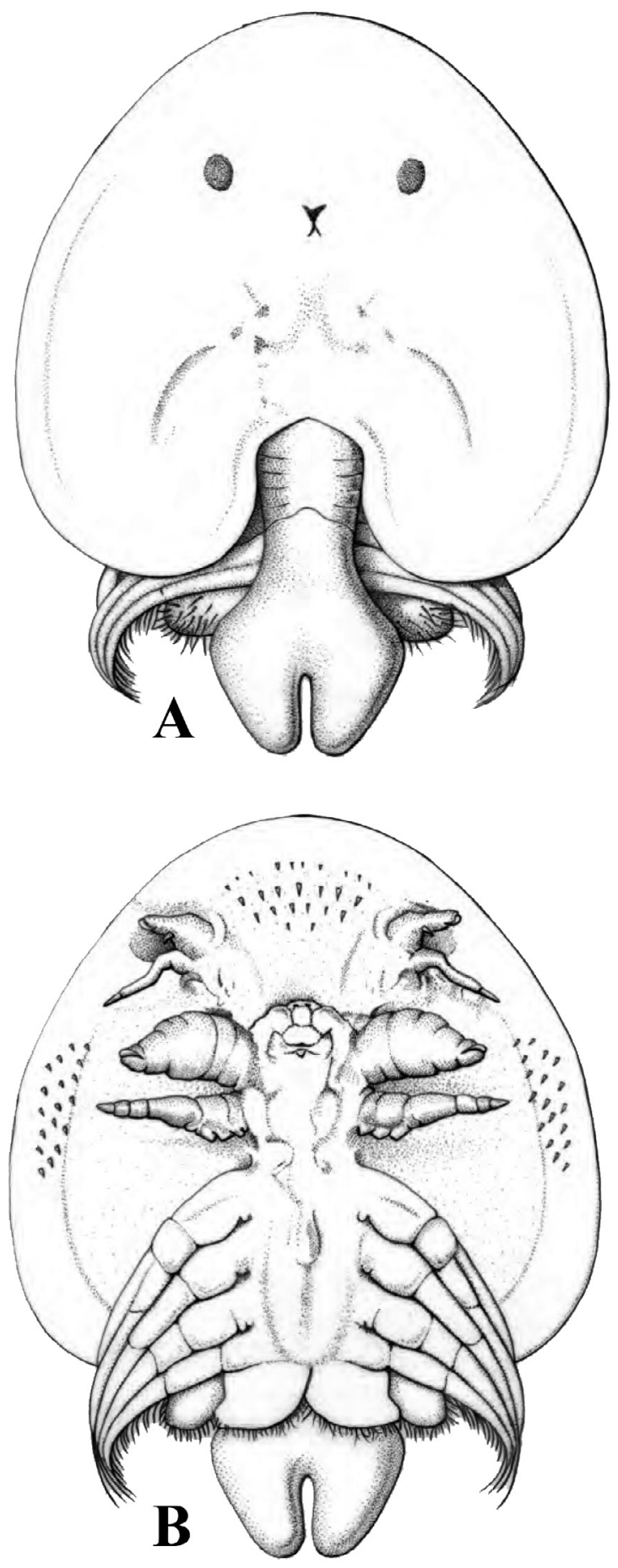

\section{$2 \mathbf{m m}$}

Figura 3. Dolops reperta (Bouvier, 1899), macho, ICN-MHN-CR 3207. A. Vista dorsal. B. Vista ventral. 
Potamotrygon schroederi es una especie más exigente en cuanto a los requerimientos de hábitat. Está asociada a aguas corrientes muy rápidas (lóticas), por lo general en el fondo del cauce, con aguas claras y alto contenido de oxígeno y en las noches pueden acercarse a las playas más someras (Lasso et al., op. cit.).

\section{Dolops striata (Bouvier, 1899) (Figura 4)}

Material examinado. Puerto Carreño, caño Dagua, departamento del Vichada, $05^{\circ} 45^{\prime} 52,4^{\prime \prime} \mathrm{N}$ - 67038'40,5"O, 28 de marzo de 2016; colectó N. Álvarez y J. Novoa, 8 machos, 1 hembra ICN-MHN-CR 3208. Hospedero: Potamotrygon schroederi (hembra adulta mantenida en cautiverio en la Estación Piscícola en Puerto Carreño).

Diagnosis. Caparazón semicircular; caparazón y abdomen cubiertos de manchas circulares de color blanco; placas basales de las segundas maxilas provistas de tres dientes; porción anterior del margen ventral con hileras de espínulas que se proyectan hasta nivel del primer par de apéndices (Thatcher, 2006).

Comentarios. Dolops striata, al igual que D. reperta, se encontró moviéndose libremente en la superficie discal (dorsal) de Potamotrygon schroederi, lo que demuestra que esta especie de raya es parasitada por las dos especies.
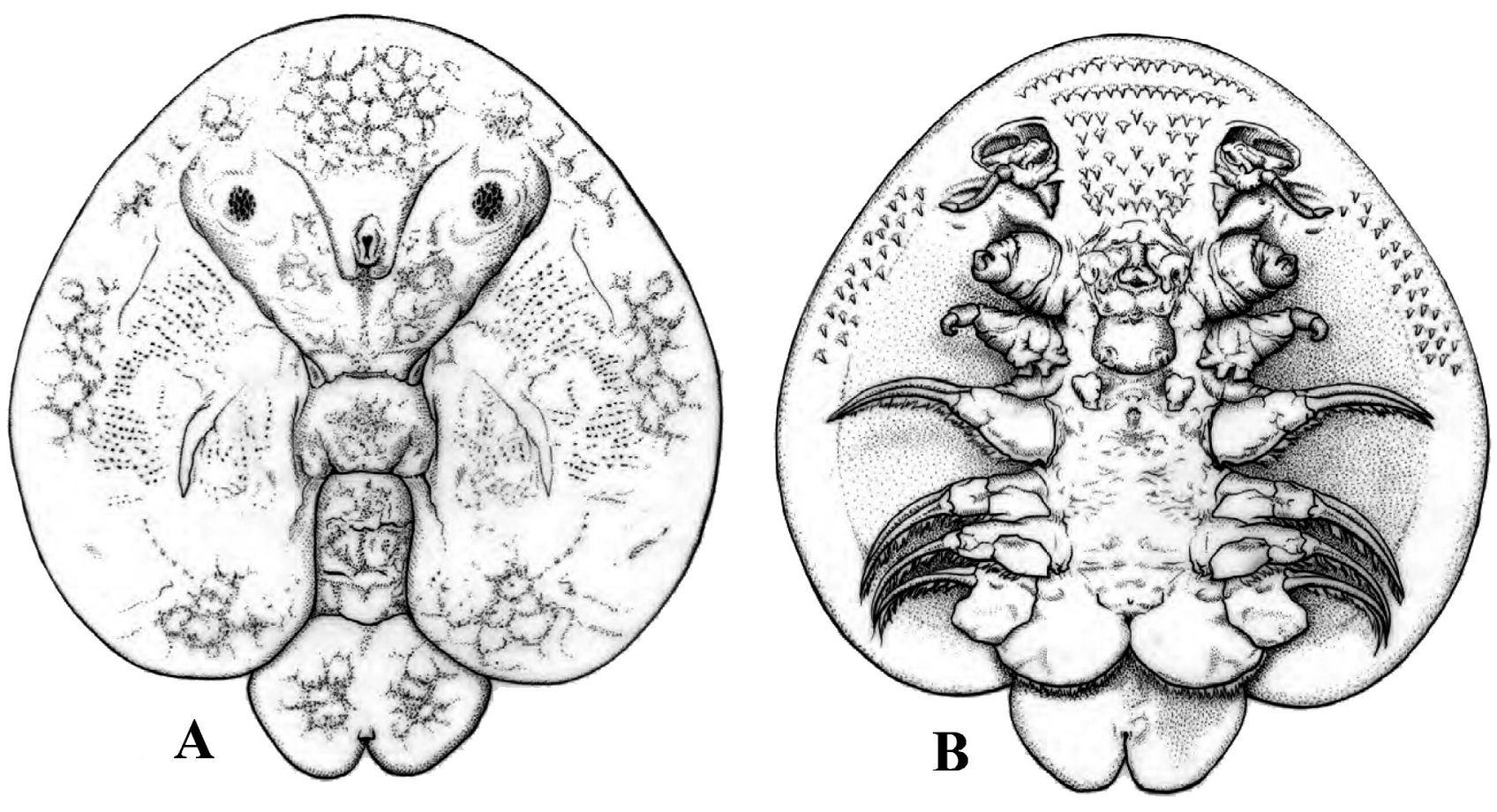

$2 \mathrm{~mm}$

Figura 4. Dolops striata (Bouvier, 1899), macho, ICN-MHN-CR 3208. A. Vista dorsal. B. Vista ventral. 


\author{
Orden Isopoda \\ Familia Cymothoidae Leach, 1818 \\ Braga Schiödte \& Meinert, 1881 \\ Braga cigarra (Szidat \& Schubart, 1960) \\ (Figura 5)
}

Material examinado. Puerto Carreño, Reserva Natural Bojonawi, laguna El Pañuelo, departamento del Vichada, $06^{\circ} 06^{\prime} 8,3^{\prime \prime} \mathrm{N}$ $74^{\circ} 02^{\prime} 21,0^{\prime \prime} \mathrm{O}, 18$ de enero de 2015, colectó Beiker Castañeda, 1 hembra con crías, ICN-MHN-CR 3209. Hospedero: Potamotrygon orbignyi, hembra adulta, $420 \mathrm{~mm}$ AD y $3 \mathrm{~kg}$ (IAvH-12829).

Diagnosis. Cefalón con porción distal ligeramente aguda; anténula más corta que la antena; pereópodos con uñas adaptadas para fijarse al hospedero; pleotelson más ancho que largo, urópodos no sobrepasan el margen posterior del pleotelson (Brandão et al., 2012).

Comentarios. Braga cigarra fue observada en la superficie ventral del disco de una hembra adulta de Potamotrygon orbignyi, adherido firmemente a la epidermis entre la cuarta y quinta abertura branquial derecha y para removerlo se utilizó una pinza. A pesar de estar firmemente adherida a la superficie ventral de la raya, no se descarta que utilice también la cámara branquial, como es lo usual en muchas de las especies de peces óseos (Thatcher, 2006). Con base en la revisión de Gama (2016), este corresponde al primer registro de parasitosis de isópodos en rayas de agua dulce.
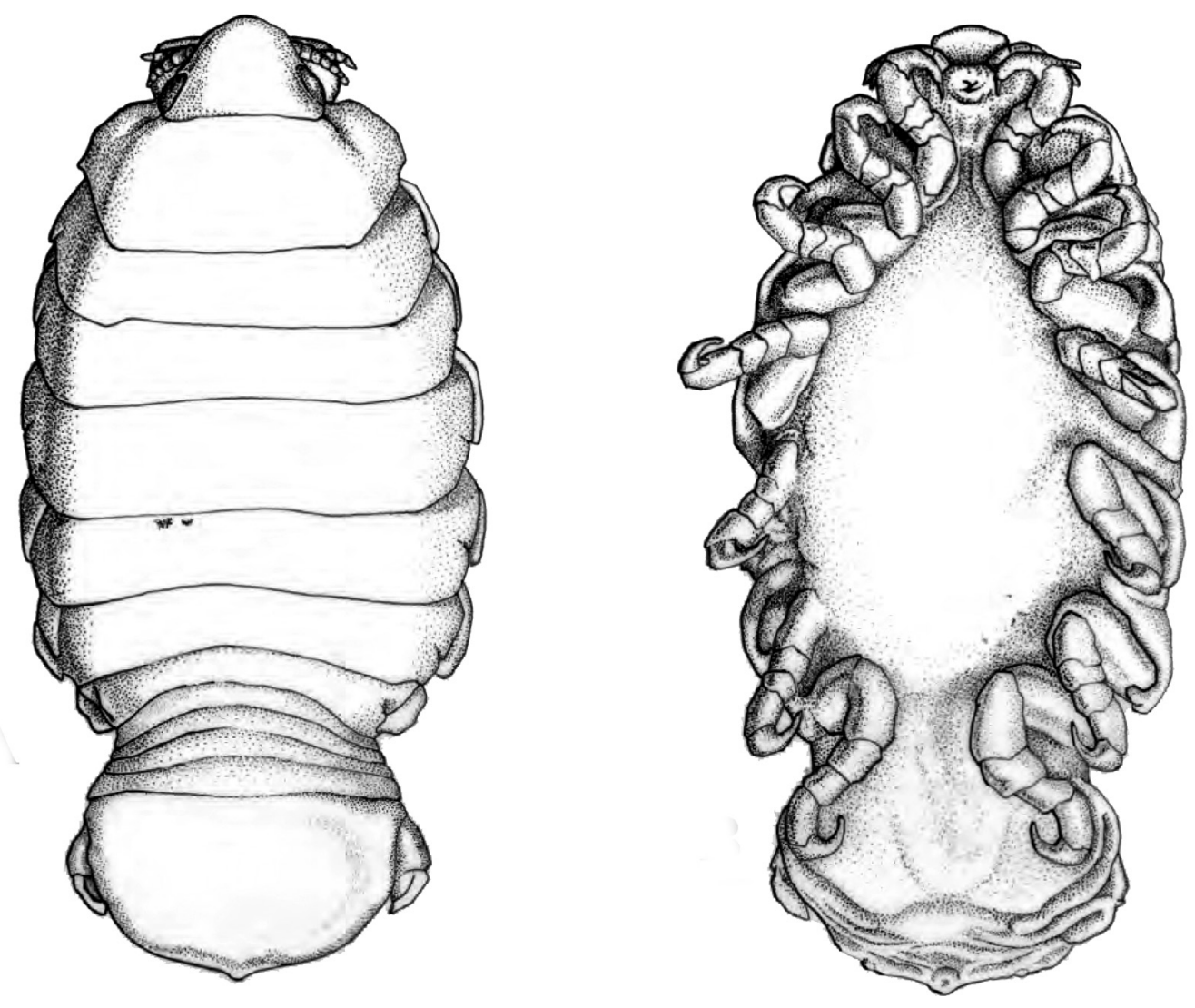

\section{$5 \mathrm{~mm}$}

Figura 5. Braga cigarra (Szidat \& Schubart 1960), hembra, ICN-MHN-CR 3209. A. Vista dorsal. B. Vista ventral. 


\section{Orden Isopoda}

Familia Corallanidae Hansen, 1890

Excorallana delaneyi Stone \& Heard, 1989

(Figura 6)
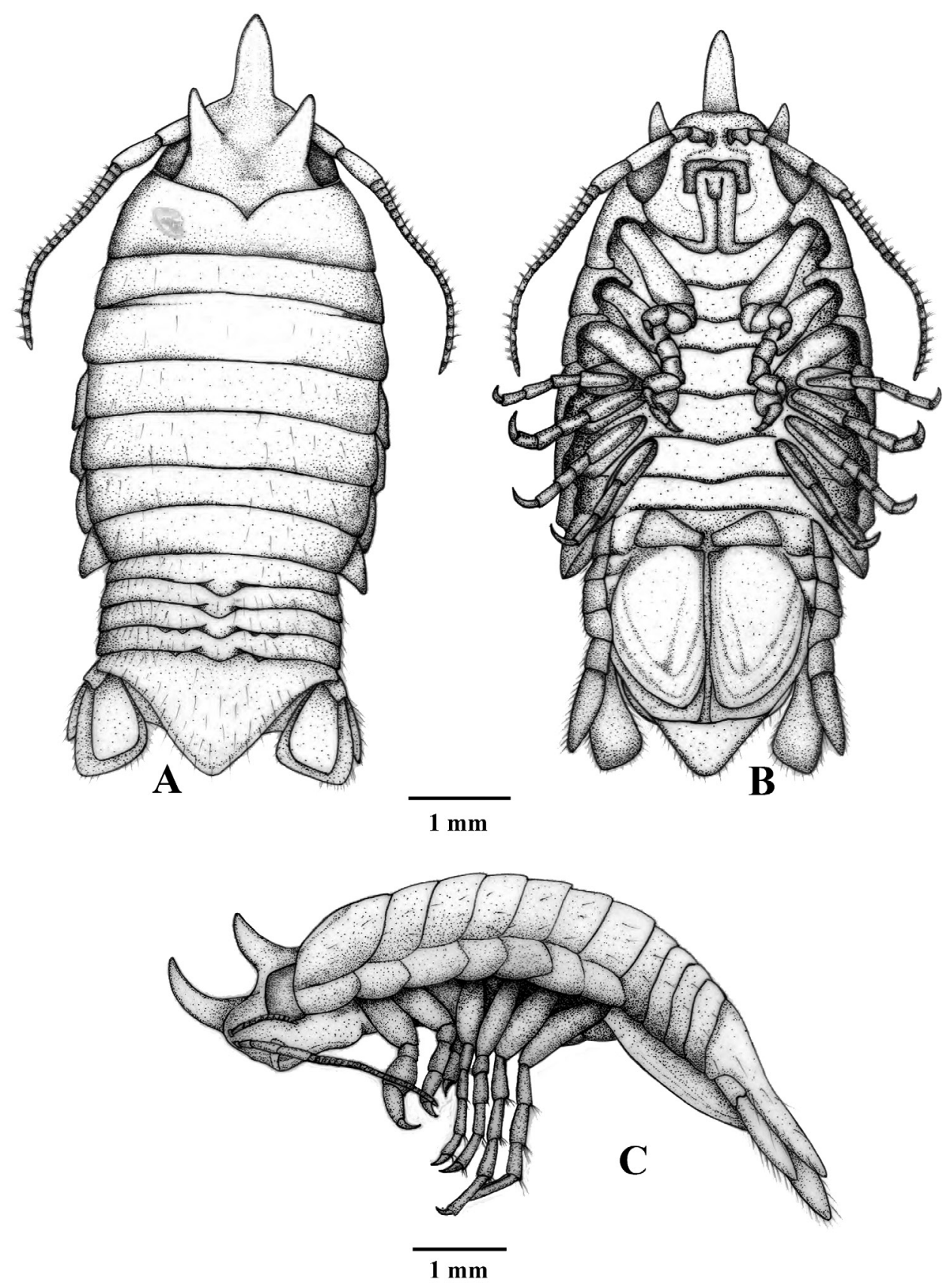

Figura 6. Excorallana delaneyi Stone \& Heard, 1989, macho, ICN-MHN-CR 3210. A. Vista dorsal. B. Vista ventral. C. Vista lateral. 
Material examinado. Puerto Carreño, caño Dagua, departamento del Vichada, $05^{\circ} 45^{\prime} 13,8^{\prime \prime} \mathrm{N}$ - 67040'9,5"W, 19 de febrero de 2017, colectó C. A. Lasso y M. A. Morales-Betancourt, ICNMHN- CR 3210; 2 machos, 3 hembras, 3 juveniles. Hospedero: Potamotrygon motoro, macho adulto $290 \mathrm{~mm}$ AD y 1,4 kg (IAvH-16142). Puerto Carreño, caño San Roque, afluente del caño Dagua, departamento del Vichada, $05^{\circ} 44^{\prime} 32,2^{\prime \prime} \mathrm{N}$ - 67040'14,3"O, 20 de febrero de 2017, colectó C. A. Lasso y M. A. Morales-Betancourt, 3 machos, 2 hembras, ICN-MHN-CR 3211. Asociados a un tronco podrido sumergido.

Diagnosis. Machos adultos con tres cuernos cefálicos, hembras adultas con un par de tubérculos; ojos grandes, separados; urópodos sobrepasan el pleotelson, pleotelson sin incisión lateral (Stone y Heard, 1989).

Comentarios. Con base en la revisión de Gama (2016) y de acuerdo con la parasitósis por Braga cigarra en Potamotrygon orbignyi, este correspondería al segundo caso de parasitosis de isópodos en rayas de agua dulce. Estos isópodos fueron colectados dentro y por fuera de los espiráculos.

\section{Discusión}

De las 350 rayas examinadas, solo en tres especímenes, de tres especies (uno por especie), se observó infestación por parásitos, lo que demuestra claramente que el nivel de infestación por especies de Dolops y los otros isópodos en el medio natural es sumamente bajo si lo comparamos con otros estudios en Brasil.

Gama et al. (2015), registraron una tasa de infestación por Argulus juparanensis en seis especies de Potamotrygon ( $\mathrm{n}=103)$, del 88,3 \% $(n=91)$, frente al $0,85 \%$ en este trabajo $(n=350)$, aunque no se descarta que durante el proceso de captura y manipulación de las rayas se hayan perdido algunos ectoparásitos, pero aun así, la tasa de infestación sigue siendo muy baja. Malta
(1983) ha observado una relación estrecha entre la estacionalidad y la tasa de infestación por argúlidos en el Amazonas, siendo mayor en la época de aguas altas en relación a la época seca. Los muestreos del presente trabajo se realizaron en la época seca, por lo que no se descarta que haya una mayor tasa de infestación en aguas altas.

Dolops reperta fue observada en Potamotrygon motoro y $P$. schroederi moviéndose libremente sobre el dorso del disco y no adherida firmemente. Weibezahn y Cobo (1964), reportan que las especies de este género se alimentan mediante desgarramientos de la epidermis del pez y la succión del plasma. La infestación por otras especies de Dolops en peces teleósteos de agua dulce y cartilaginosos del Neotrópico es bastante común (Malta, 1982, 1983; Malta y Varela, 1983; Thatcher, 1991; Mamani et al., 2004), pero es la primera vez que se registra Dolops reperta en rayas de agua dulce para la Orinoquia colombiana. No parece haber una relación de especificidad parásito-hospedero, pues $P$. schroederi resultó parasitada por dos especies de Dolops.

Por último, es importante señalar que la parasitosis aquí descrita por isópodos representan los primeros casos en rayas de agua dulce, y en el caso particular de Excorallana delaneyi, su parasitosis es facultativa.

\section{Agradecimientos}

A los colectores Natalia Álvarez-Perdomo y Pedro René Eslava (Unillanos), Jairo F. NovoaSerna (Fundación Orinoquia), Beiker Castañeda (Fundación Omacha) y Rodamir Unda. Las Fundaciones Omacha (Fernando Trujillo, Esther Marín, Jacinto Terán) y Orinoquia (Rosario Novoa, Steven Jensen), apoyaron todos los aspectos logísticos en campo. Este estudio fue financiado por el Instituto Humboldt-Programa Ciencias de la Biodiversidad. Las ilustraciones (Figuras 1 a 3) fueron elaboradas por Domingo Vega y la Figura 4 por Valentina Nieto. A los evaluadores por sus comentarios. 


\section{Referencias}

Álvarez-Perdomo, N., Eslava-Mocha, P. R. y Novoa-Serna, J. (2016). Ensayos de reproducción en cautiverio de la raya motoro Potamotrygon motoro (Müller y Henle 1841) (Potamotrygonidae) en la Fundación Orinoquia, Puerto CarreñoVichada, Colombia. Pp. 391-399. En Lasso, C. A., Rosa, R., Morales-Betancourt, M. A., GarroneNeto, D. y de Carvalho, M. R. (Eds.). XV. Rayas de agua dulce (Potamotrygonidae) de Suramérica. Parte II: Colombia, Brasil, Perú, Bolivia, Paraguay, Uruguay y Argentina. Serie Editorial Recursos Hidrobiológicos y Pesqueros Continentales de Colombia. Bogotá, Colombia: Instituto de Investigación de Recursos Biológicos Alexander von Humboldt.

Bouvier, M. E. L. (1899). Les crustacés parasites du genre Dolops Audouin (seconde partie). Bulletin de la Société Philomathique de Paris, 1: 12-40.

Brandão, H., de Melo, G., Wunderlich, A. C., Paiva, I., Carvalho, E. D. y da Silva, R. J. (2012). Ocurrence of Braga cigarra (Cymothoidae) parasitizing Galeocharax knerii (Characidae) from affluents of Jurumirim reservoir, Brazil. Revista Brasilera de Parasitologia Veterinaria, 22 (2): 292296.

Brooks, D. R. y Thorson, T. B. (1976). Two tetraphyllidean cestodes from the freshwater stingray Potamotrygon magdalenae Duméril 1852 (Chondrichthyes: Potamotrygonidae) from Colombia. The Journal of Parasitology, 62 (6): 943947.

Brooks, D. R., Mayes. M. A. y Thorson, T. B. (1979). Paravitellotrema overstreeti sp. n. (Digenea: Hemiuridae) from the Colombian freshwater stingray Potamotrygon magdalenae Duméril. Proceeding of the Helminthological Society of Washington, 46 (1): 52-54.

Brooks, D. R., Mayes, M. A. y Thorson T. B. (1981). Systematic review of cestodes infecting freshwater stingrays (Chondrichthyes: Potamotrygonidae) including four new species from Venezuela. Proceeding of the Helminthological Society of Washington, 48 (1): 43-64.
Deardoff, T. L., Brooks, D. R. y Thorson, T. B. (1981). A new species of Echinocephalus (Nematoda: Gnathostomidae) from Neotropical stingrays with comments on E. diazi. Journal of Parasitology, 67 (3): 433-439.

Domingues, M. V. y Marques, F. P. L. (2010). Phylogeny and taxonomy of Potamotrygonocotyle Mayes, Brooks y Thorson 1981 (Monogenoidea: Monocotylidae) with a descriptions of four new species. Journal of Helminthology, 1: 1-28.

Gama, C. (2016). Parasitas de raias de agua doce (Potamotrygonidae): estado actual do conhecimento e lista de espécies. Pp. 83-103. En Lasso, C. A., Rosa, R., Morales-Betancourt, M. A., Garrone-Neto, D. y de Carvalho, M. R. (Eds.). XV. Rayas de agua dulce (Potamotrygonidae) de Suramérica. Parte II: Colombia, Brasil, Perú, Bolivia, Paraguay, Uruguay y Argentina. Serie Editorial Recursos Hidrobiológicos y Pesqueros Continentales de Colombia. Bogotá, Colombia: Instituto de Investigación de Recursos Biológicos Alexander von Humboldt.

Gama, C. S., Vieira, I. M., Silva Jr., A. C. S., TavaresDias, M. y Sobrinho, A. F. (2015). Argulus juparanensis (Branchiura) em raias Potamotrygon spp. (Potamotrygonidae) na foz do rio Amazonas. Biota Amazonia, 5 (2): 134-136.

Hansen H. J. (1890). Cirolanidae et familiae nonnulae propinquae Musei Hauniensis. Det Kongelige Danske Videnskabernes Selskab Skrifter, Naturvidenskabelig og Mathematisk, 6 (3): 237-426.

Lasso, C. A., DoNascimiento, C., MoralesBetancourt, M. A. y Lasso-Alcalá, O. M. (2015). Parasitism of freshwater stingrays (Potamotrygonidae) by hematophagous catfishes (Vandelliinae). Ichthyological Exploration Freshwaters, 26 (1): 83-86.

Lasso, C. A, Morales-Betancourt, M. A., Castañeda, B., Ortiz-A., L. y Sierra-Quintero, M. T. (2016). Aspectos sobre la historia natural de las rayas de agua dulce (Potamotrygonidae) en el área de influencia de los municipios de Puerto Carreño (Vichada) e Inírida (Guainía), Orinoquia 
colombiana. Pp. 325-360. En Lasso, C. A., Rosa, R., Morales-Betancourt, M. A., Garrone-Neto, D. y de Carvalho, M. R. (Eds.). XV. Rayas de agua dulce (Potamotrygonidae) de Suramérica. Parte II: Colombia, Brasil, Perú, Bolivia, Paraguay, Uruguay y Argentina. Serie Editorial Recursos Hidrobiológicos y Pesqueros Continentales de Colombia. Bogotá, Colombia: Instituto de Investigación de Recursos Biológicos Alexander von Humboldt.

Leach, W. E. (1818). Cymothoidae. Pp. 564. En F. Cuvier (Ed.). Dictionnaire des Sciences Naturelles 12: 338-354.

Lemos, J. R. G., Oliveira, A. T., Santos, M. Q. C., Ferreira, W. R., Días, M. T. y Marcon, J. L. (2010). Índices parasitários da arraia cururu Potamotrygon cf. hystrix da bacia do médio rio Negro, Amazonas, Brasil. 62 ${ }^{\mathrm{a}}$ Reuniao Anual de SBPC.

Malta, J. C. O. (1982). Os argulídeos (Crustacea: Branchyura) da Amazonia brasileira. Aspectos da ecología de Dolops discoidalis Bouvier, 1899 e Dolops bidentata Bouvier, 1899. Acta Amazonica, 12 (3): 521-528.

Malta, J. C. O. (1983). Os argulídeos (Crustacea: Branchyura) da Amazonia brasileira 4. Aspectos da ecología de Argulus multicolor Stekhoven, 1937 e Argulus pestifer Ringuelet 1948. Acta Amazonica, 13 (3-4): 489-496.

Malta, J. C. O. y Varella, A. (1983). Os argulídeos (Crustacea: Branchyura) da Amazonia brasileira 3. Aspectos da ecología de Dolops striata Bouvier, 1899 e Dolops carvalhoi Castro, 1949. Acta Amazonica, 13 (2): 234-239.

Mamani, M., Hamel, C. y Van Damme, P. A. (2004). Ectoparasites (Crustacea: Branchiura) of Pseudoplatystoma fasciatum (surubí) and P. tigrinum (chuncuina) in Bolivian White-water floodplains. Ecología en Bolivia, 39 (2): 9-20.

Peralta, A. S. L., Carvalho Jr., J. R., Matos, E. y Serra-Freites, N. M. (1998). Ocorrencia de Argulus juparanensis Lemos de Castro 1950 (Branchiura: Argulidae) em arraia de fogo Potamotrygon motoro (Müller \& Henle, 1841) (Rajiformes: Potamotrygonidae) no Igarapé do Salito, Municipio de cachoeira do Arari, Marajó, estado do Pará. Entomología y Vectores, 5 (1): 4954 .

Schiödte, J. C. y Meinert, F. (1881). Symbolae ad Monographiam Cymothoarum Crustaceorum Isopodum, Familie II. Anilocridae. Naturhist Tidsskrift, 3 (13):1-166.

Stone, I. y Heard, R. W. (1989). Excorallana delaneyi, n. sp. (Crustacea: Isopoda: Excorallanidae) from the Northeastern Gulf of Mexico, with observations on adult characters and sexual dimorphism in related species of Excorallana, 8 (2): 199-211.

Szidat, L. y Schubart, O. (1960). Neue und seltene Parasitische süsswasser Anselm der Familie Cymothoidae aus dem River Mogi-Guassú, Brasilien (Isopoda). Anais da Academia Brasileira de Ciência, 32 (1): 107-124.

Thatcher, V. E. (1991). Amazon fish parasites. Amazoniana, 11 (3-4): 263-572.

Thatcher, V. E. (2006). Amazon Fish Parasites. Bulgaria: Ed. Pensoft, Second Edition. 508 pp.

Weibezahn, F. y Cobo, T. (1964). Seis argúlidos (Crustacea, Branchiura) parásitos de peces dulceacuícolas en Venezuela, con descripción de una nueva especie del género Argulus. Acta Biológica Venezuélica, 4 (2): 119-144. 
Carlos A. Lasso

Programa Ciencias Básicas de la Biodiversidad, Instituto de Investigación de Recursos Biológicos Alexander von Humboldt

Bogotá, Colombia

classo@humboldt.org.co

\section{Martha R. Campos}

Instituto de Ciencias Naturales,

Universidad Nacional de Colombia

Bogotá, Colombia

mhrochad@unal.edu.co

\section{Mónica A. Morales-Betancourt}

Programa Ciencias Básicas de la Biodiversidad, Instituto de Investigación de Recursos Biológicos Alexander von Humboldt

Bogotá, Colombia

mmorales@humboldt.org.co

\section{David Castro}

Instituto de Ciencias Naturales,

Universidad Nacional de Colombia

Bogotá, Colombia

dacastroba@unal.edu.co
Ectoparásitos (Argulidae, Cymothoidae, Corallanidae) en rayas de agua dulce (Potamotrygonidae) de la Orinoquia colombiana

Citación del artículo: Lasso, C. A., Campos, M. R., Morales-Betancourt, M. A. y Castro, D. (2018). Ectoparásitos (Argulidae, Cymothoidae, Corallanidae) en rayas de agua dulce (Potamotrygonidae) de la Orinoquia colombiana. Biota Colombiana, 19 (Sup. 1): 84-94. DOI: 10.21068/ c2018.v19s1a08.

Recibido: 21 de abril de 2017

Aprobado: 13 de marzo de 2018 


\section{Guía para autores \\ (http://revistas.humboldt.org.co/index.php/biota)}

\section{Envío del manuscrito}

El envío de un manuscrito implica la declaración explícita por parte del autor o los autores de que este no ha sido previamente publicado, ni aceptado para su publicación en otra revista u otro órgano de difusión científica. Todas las contribuciones son de la entera responsabilidad de sus autores y no del Instituto de Investigación de Recursos Biológicos Alexander von Humboldt, ni de la revista o sus editores.

Los trabajos pueden estar escritos en español, inglés o portugués, y no deben exceder las 40 páginas (párrafo espaciado a 1,5 líneas), incluyendo tablas, figuras y anexos. De particular interés para la revista son las descripciones de especies nuevas para la ciencia, nuevos registros geográficos, listados de especies temáticos o regionales, inventarios, bases de datos relacionados con biodiversidad, colecciones biológicas y reportes de muestreo.

Se reciben manuscritos que sean artículos científicos de investigación, así como notas de actualidad, reseñas, novedades bibliográficas y artículos de datos.

Los trabajos deben ser entregados a través del portal en línea (http://revistas.humboldt.org.co/index. php/biota) de la revista, siguiendo los pasos de registro como usuario. Todo el proceso editorial se desarrolla a través de esa plataforma.

\section{Evaluación del manuscrito}

Los manuscritos sometidos serán revisados por mínimo dos pares científicos calificados, cuya respuesta final de evaluación puede ser: a) publicado sin ningún cambio (se asume que no existe ningún cambio, omisión o adición al artículo, y que se recomienda su publicación en la forma actualmente presentada); b) aceptación condicional (se acepta y recomienda el artículo para su publicación solo si se realizan los cambios indicados por el evaluador, sean correcciones menores y no es necesaria una segunda re- visión o correcciones mayores y sí es necesaria una segunda revisión); y c) rechazo (cuando el evaluador considera que los contenidos o forma de presentación del artículo no se ajustan a los requerimientos y estándares de calidad de Biota Colombiana). Para proseguir con el proceso editorial para publicación, el manuscrito debe haber sido aceptado por el mínimo de dos pares científicos.

\section{Preparación del manuscrito}

Para la elaboración de los textos del manuscrito se debe usar un procesador de palabras (preferiblemente Word); los listados (a manera de tabla) deben ser elaborados en una hoja de cálculo (preferiblemente Excel). Para someter un manuscrito es necesario además anexar una carta de intención en la que se indique claramente:

1. Nombre completo del (los) autor (es), afiliaciones institucionales y direcciones para envío de correspondencia (es indispensable suministrar una dirección de correo electrónico para comunicación directa).

2. Título completo del manuscrito.

3. Nombres, tamaños y tipos de archivos suministrados.

4. Explicación concisa y clara, que no sobrepase tres líneas, explicando por qué el manuscrito en cuestión es ideal para que sea publicado en una revista como Biota Colombiana.

5. Lista mínimo de cuatro revisores sugeridos que puedan evaluar el manuscrito, con sus respectivas direcciones electrónicas.

**Para información sobre la preparación de un artículo de datos, continúe a la sección "Particularidades de los artículos de datos $\rightarrow$ Preparación de un artículo de datos" dentro de esta Guía de autores. 


\section{Lenguaje del manuscrito}

- Los manuscritos enviados a Biota Colombiana deben tener como requerimiento mínimo el uso adecuado del lenguaje en el que estén escritos, sea español, portugués o inglés, para asegurar la comunicación efectiva del artículo a los lectores.

- El estilo utilizado en el lenguaje del manuscrito enviado debe seguir las formalidades comúnmente aceptadas en escritos científicos y siempre buscar la claridad, concisión y cohesión en su expresión.

- Se sugiere utilizar guías de buena ortografía, redacción y estilo para el idioma en el que se escoja escribir.

\section{Especificaciones de formato}

- Para la presentación del manuscrito configure las páginas de la siguiente manera: hoja tamaño carta, márgenes de $2,5 \mathrm{~cm}$ en todos los lados, interlineado 1,5 y alineación hacia la izquierda (incluyendo título y bibliografía).

- Todas las páginas de texto deben numerarse en la parte inferior derecha de la hoja.

- Use letra Times New Roman o Arial, tamaño 12 puntos en todos los textos. Máximo 40 páginas, incluyendo tablas, figuras y anexos. Para tablas cambie el tamaño de la fuente a 10 puntos. Evite el uso de negritas o subrayados.

- Escriba los nombres científicos de géneros, especies y subespecies en cursiva (itálica). Proceda de la misma forma con los términos en latín (por ej. sensu, et al.). No subraye ninguna otra palabra o título. No utilice notas al pie de página.

- En cuanto a las abreviaturas y sistema métrico decimal, utilice las normas del Sistema Internacional de Unidades (SI) recordando que siempre se debe dejar un espacio libre entre el valor numérico y la unidad de medida (por ej. 16 $\mathrm{km}, 23{ }^{\circ} \mathrm{C}$ ). Para medidas relativas como $\mathrm{m} / \mathrm{seg}$., use m.seg-1.

- Escriba los números del uno al diez siempre con letras, excepto cuando preceden a una unidad de medida (por ej. $9 \mathrm{~cm}$ ) o si se utilizan como marcadores (por ej. parcela 2, muestra 7). Los números mayores a diez deben ser escritos con los símbolos numéricos arábigos. Si en el mismo párrafo se utilizan cifras menores a diez y cifras mayores a diez, se deben unificar para dejar las cifras solo con símbolos numéricos arábigos.

- No utilice punto para separar los millares, millones, etc (por ej. 54000). Utilice la coma para separar en la cifra la parte entera de la decimal (por ej. 3,1416) cuando el texto es en español. En el caso del inglés, los decimales se separan con puntos (por ej. 3.1416). Enumere las horas del día de 0:00 a 24:00.

- Exprese los años con todas las cifras sin demarcadores de miles (por ej. 1996-1998). En español los nombres de los meses y días (enero, julio, sábado, lunes) siempre se escriben con la primera letra minúscula, en inglés se escriben con la primera letra mayúscula (January, July, Saturday, Monday).

- Los puntos cardinales (norte, sur, este y oeste) en español siempre deben ser escritos en minúscula, a excepción de sus abreviaturas N, S, E, O (en inglés $W$ ), y cuando son referidos como puntos o hacen parte de un nombre propio (p.e. cordillera Oriental). La indicación correcta de coordenadas geográficas es como sigue: $02^{\circ} 37^{\prime} 53^{\prime \prime} \mathrm{N}-$ $56^{\circ} 28^{\prime} 53^{\prime \prime} \mathrm{O}$. La altitud geográfica se citará como se expresa a continuación: 1180 m s.n.m. y en inglés $1180 \mathrm{~m}$ a.s.l.

- Las abreviaturas se deben explicar la primera vez que son usadas.

- Al citar las referencias en el texto, siga las normas APA (Manual de Publicaciones de la American Psychological Association, Sexta Edición). Incluya los apellidos de los autores en caso de que sean uno o dos, y el apellido del primero seguido por et al. (en cursiva) cuando sean tres o más. En el caso de dos autores, los apellidos deben ser separados por la palabra "y" (por ej. Cochran y Goin, 1970). En inglés, los apellidos deben estar separados por " $\&$ ". Si menciona varias referencias, éstas deben ser ordenadas cronológicamente y separadas por punto y coma (por ej. Rojas, 1978; Bailey et al., 1983; Sephton, 2001, 2001). Inserte una coma después de los nombres de los autores y antes del año de la referencia (Acevedo, 2009). 
- Las referencias que son autoridad taxonómica de descripción no deben ser incluidas en la lista de referencias al final pero sí en el texto.

- Refiera las figuras (gráficas, diagramas, ilustraciones y fotografías) sin abreviación (por ej. Figura 3) al igual que las tablas (por ej. Tabla 1). Gráficos y figuras deben presentarse con tipo y tamaño de letra uniforme.

- Las figuras deben ser nítidas y de buena calidad, evitando complejidades innecesarias (por ej. tridimensionalidad en gráficos de barras, marcos o efectos tridimensionales); si es posible use solo colores sólidos en lugar de tramas. Las letras, números o símbolos de las figuras deben ser de un tamaño adecuado de manera que sean claramente legibles una vez reducidas.

- Cada figura debe estar insertada en el texto, y adicionalmente se debe enviar su archivo aparte en alta calidad en el paso de "Cargar los archivos complementarios". Para el caso de las fotografías y figuras digitales es necesario que estas sean guardadas como formato tiff, jpg o png con una resolución de 300 dpi.

- Las tablas y anexos deben ser simples en su estructura (marcos) y estar unificados. Haga las llamadas a pie de página de tabla con letras ubicadas como superíndice. Evite tablas grandes sobrecargadas de información y líneas divisorias o presentadas en forma compleja.

\section{Secciones del manuscrito}

** Para información sobre las secciones de un artículo de datos, continúe a la sección "Particularidades de los artículos de datos $\rightarrow$ Secciones de un artículo de datos" dentro de esta Guía de autores.

- Los manuscritos deben llevar el siguiente orden: título, resumen y palabras clave, abstract y keywords, introducción, materiales y métodos, resultados, discusión, conclusiones (optativo), agradecimientos (optativo) y referencias. Seguidamente, presente una página con la lista de tablas, figuras y anexos.

- Las secciones del manuscrito en el texto deben ir en negrilla, con solo la primera letra en mayúscula. Si necesita agregar subtítulos a las secciones, estos deben ir en la misma línea en la que comienza el texto del párrafo, separados por un punto del resto del texto, y en negrilla.

Título: conciso y explicativo, debe informar sobre el contenido del manuscrito.

Resumen: da un resumen de máximo 200 palabras sobre el manuscrito, en el que se debe incluir el objetivo, métodos, resultados y conclusiones principales del manuscrito. Si se presenta algo que sea novedoso o excepcional, se debe hacer mención aquí. El resumen se debe escribir en dos idiomas (español o portugués y abstract en inglés).

Palabras clave: máximo cinco palabras clave, complementarias al título del artículo, en español o portugués e inglés, separadas por un punto entre cada término. Deben presentarse en orden alfabético. Se sugiere el uso de tesauros temáticos para encontrar sinónimos y términos adecuados.

Introducción: presenta el tema y da el contexto necesario para el desarrollo del manuscrito. El propósito u objetivo principal del trabajo debe hacerse explícito en esta sección.

Materiales y métodos: hace una descripción detallada del procedimiento, incluyendo los materiales, lugar, fechas, métodos estadísticos, etc. que se utilizaron en el trabajo. Debe ser lo suficientemente completo para que otros investigadores puedan replicar el trabajo y si se usa una metodología novedosa debe explicarse y sustentarse.

Resultados: presenta los hallazgos del trabajo de manera organizada y con uso adecuado de figuras. Evitar la inclusión de tablas muy extensas en esta sección y más bien incluir como anexos si es el caso.

Discusión: se destacan los puntos más relevantes, polémicos o novedosos del trabajo y se explican los resultados principales en relación a la importancia o aportes del trabajo en su área.

Conclusiones: reflexiones finales sobre el trabajo con relación a su propósito y objetivos, frecuentemente direccionando hacia acciones e investigaciones futuras.

Agradecimientos: Párrafo sencillo y conciso entre el texto y la lista de referencias. Mencione fuentes 
de financiación o apoyo que recibió el proyecto. Evite títulos como Dr., Lic., TSU, etc.

Referencias: La revista sigue las normas de citación APA (Manual de Publicaciones de la American Psychological Association, Sexta Edición). La lista de las referencias contiene únicamente aquellas citadas en el texto. Ordénelas alfabéticamente por autores y cronológicamente para un mismo autor. Si hay varias referencias de un mismo autor(es) en el mismo año, añada las letras a, b, c, etc. al año. No abrevie los nombres de las revistas. Incluya todos los autores de la referencia. Presente las referencias al final del manuscrito.

\section{Ejemplos de citación}

\section{Artículo en revistas:}

Antonelli, A., Nylander, J. A., Persson, C. y Sanmartín, I. (2009). Tracing the impact of the Andean uplift on Neotropical plant evolution. Proceedings of the National Academy of Sciences, 106(24): 9749-9754.

\section{Libros:}

Gutiérrez, F. P. (2010). Los recursos hidrobiológicos $y$ pesqueros en Colombia. Bogotá: Instituto de Investigación de Recursos Biológicos Alexander von Humboldt. 118 pp.

\section{Tesis:}

Cipamocha, C. A. (2002). Caracterización de especies y evaluación trófica de la subienda de peces en el raudal Chorro de Córdoba, bajo río Caquetá, Amazonas, Colombia. (Trabajo de grado). Bogotá D. C.: Universidad Nacional de Colombia, Facultad de Ciencias, Departamento de Biología. 160 pp.

\section{Informes técnicos:}

Andrade, G. I. (2010). Gestión del conocimiento para la gestión de la biodiversidad: bases conceptuales y propuesta programática para la reingeniería del Instituto Humboldt. (Informe técnico). Bogotá D. C.: Instituto de Investigación de Recursos Biológicos Alexander von Humboldt. 80 pp.

\section{Capítulo en libro o en informe:}

Fernández F., Palacio, E. E. y MacKay, W. P. (1996). Introducción al estudio de las hormigas
(Hymenoptera: Formicidae) de Colombia. En Amat, G. D., Andrade, G. y Fernández, F. (Eds.). Insectos de Colombia. Estudios Escogidos. Pp: 349-412. Bogotá: Academia Colombiana de Ciencias Exactas, Físicas y Naturales y Centro Editorial Javeriano.

\section{Resumen en congreso, simposio, talleres:}

Señaris, J. C. (2001). Distribución geográfica y utilización del hábitat de las ranas de cristal (Anura; Centrolenidae) en Venezuela. Trabajo presentado en Programa y Libro de Resúmenes del IV Congreso Venezolano de Ecología, Mérida, Venezuela. p. 124.

\section{Ley o Decreto:}

Congreso de Colombia. (8 de febrero de 1994) Ley General de Educación. [Ley 115 de 1994]. DO: 41.214.

\section{Páginas web:}

No serán incluidas en la lista de referencias, sino que se señalan claramente en el texto al momento de mencionarlas.

\section{Particularidades de los artículos de datos}

Un Artículo de Datos o Data Paper es un tipo de publicación académica que surgió como un mecanismo para incentivar la publicación de datos sobre biodiversidad. Es un medio para generar reconocimiento académico y profesional adecuado a todas las personas que intervienen, de una manera u otra, en la gestión de información sobre biodiversidad, y además sirve para destacar la existencia y relevancia de los conjuntos de datos frente el resto de la comunidad científica.

Como su nombre lo sugiere, este tipo de artículos se basan en la descripción de un conjunto de datos primarios, y aunque no es una investigación científica sensu stricto, se espera que contengan información acerca de la historia del conjunto de datos (propósito del mismo, metodología sobre la toma de los datos, financiadores, coberturas taxonómicas y geográficas, etc.) y sobre su valor y utilidad (básica o aplicada) para la comunidad científica (Chavan y Penev, 2011) ${ }^{1}$. Lo novedoso y ventajoso de este mo-

\footnotetext{
${ }^{1}$ Chavan, V. y Penev, L. (2011). The data paper: The mechanism to incentivize data publishing in biodiversity science. BMC Bioinformatics 2011, 12(Sup. 15): S2
} 
delo de publicación es que el manuscrito siempre está vinculado al conjunto de datos, a través de un enlace a un repositorio web persistente y confiable, el IPT (Integrated Publishing Toolkit). Adicionalmente los metadatos que describen ese conjunto de datos y que están documentados en la misma herramienta, deben citar el artículo de datos.

Se recomienda someter un artículo de datos, cuando los datos a los que hace referencia son primarios, originales y están restringidos temporal y metodológicamente, se encuentran disponibles en agregadores de datos como el SiB Colombia y GBIF, y pueden ser estructurados con el estándar Darwin Core (DwC) como en el caso de:

- Observaciones de un proyecto particular

- Colecciones biológicas

- Listados de especies

- Datos genómicos

- Eventos de muestreo

- Inventarios

- Bases de datos

- Rasgos funcionales

Los conjuntos de datos que no cumplan estas características, no serán aceptados para publicación como artículo de datos. Tal es el caso de compilaciones de registros biológicos de fuentes secundarias (por ej., literatura).

\section{Preparación de un artículo de datos (publicación de datos y creación del manuscrito)}

Un artículo de datos busca describir todos los tipos de recursos de datos sobre biodiversidad. De esta manera, el artículo de datos siempre está enlazado al conjunto de datos que describe a través de una URL o DOI.

A continuación encontrará el procedimiento de cómo generar y someter un manuscrito para artículo de datos usando las herramientas y modelo de publicación del SiB Colombia. Cabe resaltar que la revista también recibe artículos de datos que tengan conjuntos de datos publicados en otras plataformas reconocidas, siempre y cuando cuenten con los requisitos de ser repositorios confiables y tengan un enlace IPT disponible. Igualmente, las secciones de los manuscritos enviados deben seguir las enunciadas en la Tabla 1.

Los artículos de datos, como los demás tipos de manuscritos enviados a la revista, serán sometidos a evaluación por pares y deben cumplir las mismas especificaciones de formato, normas de citación y uso del lenguaje, además de incluir una carta de intención, como se menciona en esta Guía de autores. Adicionalmente, durante la evaluación del manuscrito, los datos descritos deben estar disponibles en línea de manera abierta en un repositorio público y con una licencia robusta de atribución y uso.

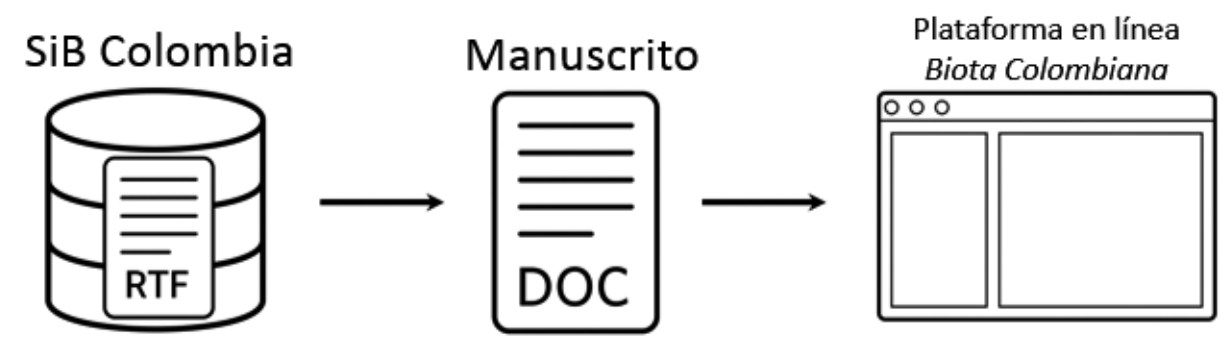

Figura 1. Proceso general de sometimiento de un artículo de datos desde el SiB Colombia a la revista Biota Colombiana. 
Paso 1.

Publicación de los datos a través del SiB Colombia

El SiB Colombia cuenta con un modelo de publicación de datos que hace uso del IPT como herramienta. Desde el IPT, es posible generar una primera versión del manuscrito a partir de los metadatos en formato de texto enriquecido (RTF), siempre $y$ cuando el conjunto de datos respectivo ya haya sido indexado por el SiB Colombia y cuente con los metadatos suficientes (el proceso de publicación de datos a través del SiB Colombia puede ser consultado en https://www.sibcolombia.net/).

A. Registro de organización. Para poder publicar a través del SiB Colombia, es necesario que la organización esté registrada como socio publicador. Se puede comprobar en este enlace si ya es así. En caso contrario, es muy sencillo hacer parte de la red de socios, solo se debe diligenciar el Formulario de Registro.

B. Estandarización de datos. Los datos se deben estructurar en una tabla plana haciendo uso del estándar Darwin Core (DwC). Para esto, se puede descargar la plantilla que se adapte a el tipo de datos o generar una plantilla a través del Generador de Plantillas Excel.

C. Calidad de sus datos. Se debe verificar y mejorar la calidad de los datos haciendo uso de herramientas para identificar, limpiar y corregir posibles errores geográficos, taxonómicos o de formato, entre otros.

D. Cargar datos en línea. El IPT es una herramienta que facilita compartir diferentes tipos de datos relacionados con la biodiversidad siempre y cuando estos se encuentren estructurados según el estándar DwC. Para cargar datos en el IPT, debe contar con una cuenta de usuario en uno de los IPT disponibles por el SiB Colombia. Si aún no se tiene una cuenta, puede contactar al Equipo Coordinador del SiB Colombia (EC-SiB) y solicitarla a través del correo electrónico sib@humboldt.org.co.

E. Mapear datos. Una vez se haya cargado el conjunto de datos, se debe verificar que estén siendo leídos en correspondencia con los elementos DwC. El manual de usuario del IPT está disponible para más información o se puede contactar al EC-SiB.
F. Creación de metadatos. La estructura de los metadatos se parece en gran medida a un artículo de investigación tradicional. Estos metadatos mantienen la estructura general de un artículo de datos y facilitan la generación del mismo. En la sección Metadatos del IPT, se debe documentar toda la información que permite dar un contexto a los datos. En total hay 12 secciones para ingresar información que describe el conjunto de datos. El manual de usuario del IPT está disponible para más información o se puede contactar al EC-SiB.

G. Publicar el recurso y notificar al EC-SiB. Una vez completados los pasos anteriores, el IPT activará la opción 'Publicar'. Se debe hacer clic en este botón y enviar un correo a sib@humboldt.org.co para notificar al EC-SiB de su publicación. El correo debe tener como asunto "Recurso publicado" y contener:

- Nombre

- Nombre de organización

- Nombre del recurso publicado

- URL de la vista general del recurso después de publicado

En este punto los datos serán indexados por el SiB Colombia y GBIF, y contarán con un identificador persistente DOI.

\section{Paso 2}

Generación del manuscrito para artículo de datos usando el IPT

El IPT en el cual ha sido publicado el conjunto de datos permite generar un manuscrito RTF que describe al conjunto de datos. El enlace al conjunto de datos aparecerá en el manuscrito bajo el título "Data published through GBIF". A continuación se describe el paso a paso para generar el manuscrito desde los metadatos del conjunto de datos publicado a través del SiB Colombia.

- En la página principal del recurso publicado a través del IPT, se debe hacer clic sobre el botón RTF para descargar una versión del manuscrito en texto enriquecido que se puede abrir en cualquier procesador de texto (por ej: Word) (Figura 2). 


\section{Escarabajos coprófagos de la cuenca alta y media del río Bita, Vichada} (Colombia)

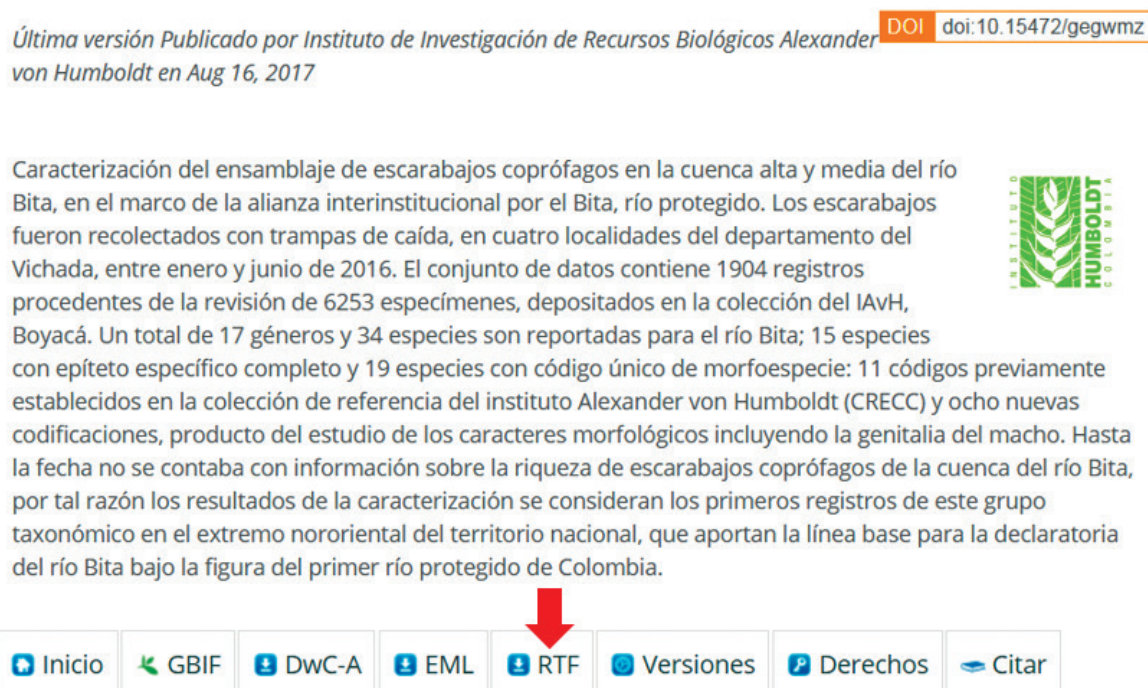

Figura 2. Los metadatos de una conjunto de datos, pueden ser descargados del IPT como archivo RTF, la primera versión del manuscrito para someter a la revista.

- El manuscrito descargado se encuentra en inglés. Los ajustes necesarios de acuerdo a los lineamientos de la revista Biota Colombiana deben ser realizados y la plantilla para artículos de datos se puede descargar aquí.

- Una vez ajustado el manuscrito con los textos adicionales, tablas y figuras, puede ser sometido a evaluación a través del portal en línea de la revista, siguiendo los pasos de registro como usuario. Todo el proceso editorial se desarrolla a través de esa plataforma.

\section{Paso 3}

\section{Ajustes y correcciones del manuscrito para artículos de datos}

Cuando el manuscrito ha sido sometido a evaluación, este se somete a revisión por pares evaluadores de acuerdo a los lineamientos establecidos por la revista para evaluadores de artículos de datos.

Después de evaluado, y en caso de ser aceptado, el manuscrito será devuelto al autor con los comentarios de los revisores y del editor de la revista con el objetivo de realizar las modificaciones antes de publicar. Como autor, deberá realizar todas las correcciones o adiciones recomendadas directamente en los metadatos del IPT y no en el manuscrito del artículo de datos. De esta forma se mejoran también los metadatos del conjunto de datos sometido.

Una vez se hayan mejorado los metadatos en el IPT, se debe actualizar la publicación del recurso para que los cambios se vean reflejados. En la página principal del recurso publicado a través del IPT, ubique el botón RTF y haga clic sobre el mismo para descargar una versión mejorada del manuscrito en texto enriquecido que puede abrir nuevamente en cualquier procesador de texto (por ej. Word).

Después de la re-inserción manual de los textos adicionales y asegurarse que esta versión cumple con los requerimientos de la revista, el manuscrito debe ser enviado nuevamente a la revista.

\section{Secciones de un artículo de datos}

A diferencia de los otros tipos de manuscritos que pueden ser sometidos a la revista Biota Colombiana, los artículos de datos incluyen las secciones estipuladas en la Tabla 1. 
Tabla 1. Estructura del artículo de datos y su correspondencia con los elementos del GMP incorporados en el IPT.

\section{Correspondencia con los elementos del IPT}

Nombre de la sección

\section{Título}

Autores

Afiliaciones

Autores de contacto
Derivado del elemento Título. Centrado sin punto al final.

Derivado de los elementos Creador del recurso, Proveedor de los metadatos y Partes asociadas. De estos elementos, se deriva la combinación nombre y apellido separados por comas. Las afiliaciones de los autores se indican con números $(1,2,3 \ldots)$ al final de cada apellido como superíndice. Centrado.

Derivado de los elementos Creador del recurso, Proveedor de los metadatos y Partes asociadas. De estos elementos, la combinación de organización, dirección, código postal, ciudad, país y correo electrónico, constituyen la afiliación. Si dos o más autores comparten la misma afiliación, se indica con el mismo número.

Derivado de los elementos Creador del recurso y Proveedor de los metadatos. De estos elementos, se deriva la combinación nombre, apellido y correo electrónico. Los correos electrónicos son escritos en paréntesis. Si hay más de un autor como contacto, estos van separados por comas. Si el Creador del recurso y Proveedor de los metadatos es el mismo autor, el Creador del recurso se refleja como el autor de contacto. Texto centrado.

Insertadas manualmente por el Editor Asistente de la revista para indicar las fechas de presentación original del manuscrito, revisión, aceptación y publicación como un artículos de datos en Biota Colombiana.

Fechas de recibido, revisado, aceptado y publicado

\section{Resumen}

Derivado del elemento descripción. El resumen debe incluirse tanto en español o portugués como inglés.

Derivadas del elemento palabras claves. Las palabras van separadas por comas. Las palabras deben estar escritas tanto en español o portugués como inglés.

Palabras Clave

Introducción No se deriva de ningún elemento del GMP y debe ser adicionada manualmente.

Cobertura taxonómica

Derivada de los elementos de la sección cobertura taxonómica: descripción, nombre científico, nombre común y categoría.

Cobertura geográfica

Derivada de los elementos de la sección cobertura geográfica: descripción, latitud mínima, latitud máxima, longitud mínima, longitud máxima.

Cobertura temporal

Derivada de los elementos de la sección cobertura temporal: fecha inicial y fecha final.

Derivada de los elementos de la sección datos del proyecto: título, persona del proyecto, fuentes de financiación, descripción del área de estudio y descripción del diseño.

Descripción del proyecto

Derivada de los elementos de la sección datos de la colección: nombre de la colección,

Descripción de la colección biológica

identificador de la colección, identificador de la colección parental, método de preservación de los especímenes y unidades curatoriales.

Derivado de los elementos de la sección métodos de muestreo: área de estudio, descripción del muestreo, control de calidad, descripción del paso metodológico.

\section{Materiales y métodos}

\section{Resultados}

$--$

Derivado de los elementos de la sección enlaces externos entre otros: nombre, conjunto de caracteres, URL del archivo, formato del archivo, versión del formato del archivo, fecha de publicación, idioma, derechos de propiedad intelectual. Puede adicionar manualmente una descripción adicional de los datos como texto, figuras y tablas.

Información adicional

Derivado del elemento información adicional.

Discusión

No se deriva de ningún elemento del GMP y debe ser adicionada manualmente, enfocada en el potencial de uso de los datos en investigación, educación o toma de decisiones.

Agradecimientos

Referencias

No se deriva de ningún elemento del GMP y debe ser adicionada manualmente.

Derivado del elemento referencia en bibliografía. 


\section{Guidelines for authors}

\section{(http:// revistas.humboldt.org.co/index.php/biota)}

\section{Submitting a manuscript}

Submitting a manuscript implies the explicit statement by the author(s) that the paper has not been published before, nor accepted for publication in another journal or other means of scientific diffusion. Contributions are entire responsibility of the author(s) and not the Research Institute of Biological Resources Alexander von Humboldt, or the journal and their editors.

Papers can be written in Spanish, English or Portuguese, and should not exceed the maximum length of 40 pages (with paragraph lines spaced at 1.5) including tables, figures and appendices. Of particular interest for this journal are descriptions of new species for science, new geographic records, thematic or regional species lists, inventories, databases related to biodiversity, biological collections and sampling reports.

Biota Colombiana receives scientific research articles, as well as notes, reviews, bibliographic novelties and data papers.

Manuscripts must be submitted through the online platform of the journal (http://revistas.humboldt. org.co/index.php/biota) as a registered user. The complete editorial process is managed in this platform.

\section{Evaluation}

Submitted manuscripts will be reviewed by at least two qualified scientific peers. Results of the peer revisions may include any of the following: a) accepted (in this case it is assumed that no change, omission or addition to the article is required and may be published as presented.); b) conditional acceptance (the article is accepted and recommended for publication only if indicated corrections are made; corrections may be minor and a second evaluation is not necessary or major and a second evaluation is necessary); and c) rejected (reviewer considers that the contents and/or form of the paper are not in accordance with requirements of publication standards of Biota Colombiana). For a manuscript to continue its editorial process, it must have been accepted by at least two reviewers.

\section{Preparation of Manuscript}

Any word-processor program may be used to write the text of the manuscript (Word is recommended). Lists or any other type of table must be presented in spreadsheets (Excel is recommended). To submit a manuscript, a cover letter that clearly indicates the following must be sent:

1. Full names, institutional filiations, and e-mail addresses of all authors. (Please note that email addresses are essential to direct communication)

2. Complete title of the article

3. Names, sizes and types of files provided.

4. Concise and clear sustentation of why the presented manuscript is in concordance with the type of articles published in the journal. Such explanation must not surpass a maximum of three lines.

5. List of the names and e-mail addresses of at least four peers who are qualified to review the manuscript.

\footnotetext{
**For information regarding the preparation of data papers, continue to "Details for Data Papers-> Preparation of Data Paper"
} 


\section{Use of Language}

- Manuscripts that are sent to Biota Colombiana have as a minimum requirement for consideration the appropriate use of language in writing, regardless if they are presented in Spanish, Portuguese or English.

- The style should follow the common formalities of scientific writing and be clear, concise and cohesive.

- The use of guides for correct spelling, grammar and style is recommended.

\section{Format}

- Texts must follow the format of standard letter size paper, with $2.5 \mathrm{~cm}$ margins on all sides, 1.5-spaced and left-aligned paragraphs (including title and bibliography).

- All pages must be numbered in the lower right corner.

- Font must be Times New Roman or Arial, size 12, in all parts of the text, except tables (size 10). Manuscript must not exceed a maximum length of 40 pages, including tables, figures and appendices. Avoid the use of bold or underlined font.

- Scientific names of genera, species and subspecies must be in italics, as well as Latin technical terms (i.e sensu, et al.). Avoid underlining any word or title. Do not use footnotes.

- For abbreviations and the metric system, use the standards of the International System of Units (SI). Leave a space between the numeric value and the measure unit (p.e. $16 \mathrm{~km}, 23{ }^{\circ} \mathrm{C}$ ). For relative measures such as $\mathrm{m} / \mathrm{sec}$, use $\mathrm{m} . \mathrm{sec}-1$.

- Write the numbers between one to ten in letters except when it precedes a measure unit (p.e. $9 \mathrm{~cm}$ ) or is used as a marker (p.e. lot 2, sample 7). Numbers greater than ten must be written in Arabic numerals. If in the paragraph both numbers lesser than ten and greater than ten appear, all should be written in Arabic numerals.

- Thousands, millions, etc. should not be separated by commas nor periods (p. e. 54000). Use periods to separate decimals (p. e. 3.1416). In Spanish, use commas to separate decimals (p. e. 3,1416). Hours should be represented in military time from 0:00 to $24: 00$.

- Years should be written without commas or periods (p. e. 1996-1998). In English months and days of the week are capitalized (January, July, Saturday, Monday), whereas in Spanish the first letter of months and days of the week are written with lower-case letters (enero, julio, sábado, lunes).

- Cardinal points (north, south, east and west) must be written in lower case letters, and only be capitalized if used in abbreviation N, S, E, W (O in Spanish) or as part of a proper name ( $p$. e. North Carolina). Correct use of coordinates is: $02^{\circ} 37^{\prime} 53^{\prime \prime} \mathrm{N}-56^{\circ} 28^{\prime} 53^{\prime \prime} \mathrm{O}$. Altitude should be expressed as $1180 \mathrm{~m}$ a.s.l. and $1180 \mathrm{~m}$ s.n.m. in Spanish.

- All abbreviations must be explained the first time they are used.

- When citing in-text references, follow APA citation norms (American Psychological Association Publications Manual, Sixth Edition). Include last names of authors if there are only one or two authors, or the first author followed by et al. (in italics) if there are three or more authors. In the case of two authors, last names must be separated by "\&" (p. e. Cochran \& Goin, 1970). In Spanish, last names of two authors must be separated by " $y$ ". If many references are cited, they must be ordered chronologically and separated by semicolons (p. e. Rojas, 1978; Bailey et al., 1983; Sephton, 2001, 2001). Notice that a comma must be inserted after the authors and before the year (Acevedo, 2009).

- Taxonomic references must not be included in the final list of Literature cited, but they must appear in the text of the manuscript.

- Refer to all figures (graphs, diagrams, illustrations, photos) and tables without abbreviation (p. e. Figure 3, Table 1). All figures and tables should have uniformity in font and letter size. 
- Figures must be clear and have a good quality. Unnecessary complexities (such as 3D effects, frames, etc.) should be avoided. If possible, only use solid colors instead of textures. Letters, numbers or symbols must be in legible sizes.

- All figures must be inserted in the text of the manuscript and sent in a separate file in high quality in the step of "Charge complementary files". For photos and digital figures, files must be in tiff, jpg or png format in a resolution not lower than $300 \mathrm{dpi}$.

- Tables and appendices must have a simple and uniform structure. Footnotes in tables must be as superscript letters. Avoid extensive tables with too much information and dividing lines.

\section{Parts of the Manuscript}

** For information about the parts of data papers, continue to "Details for Data Papers -> Parts of Data Paper".

- Submitted manuscripts must contain the following sections: title, abstract and keywords in English and Spanish, Introduction, Materials and methods, Results, Discussion, Conclusions (optional), Acknowledgements (optional) and Literature cited. At the end of the manuscript, include a list with the tables, figures and appendices.

- Sections subtitles must be written in bold, with only the first letter capitalized. If sections have subtitles, they must be written in bold in the first line of the paragraph, separated by a period from the beginning of the paragraph.

Title: concise and explanatory, must clearly communicate what will be found in the article.

Abstract: a summary of the article, with a maximum length of 200 words. It must include the objectives, methods, results and major conclusions of the study. If the article has a novel or extraordinary finding, it must be highlighted in this section. Abstracts must be written in two languages: Spanish or Portuguese, and English.
Keywords: up to five keywords. They must be complementary to the title (not repeated) and written in Spanish or Portuguese, and English. Words must be separated by periods and presented in alphabetical order. The use of thesaurus to find appropriate synonyms is recommended.

Introduction: presentation of the topic, with enough context to support the rest of the article. The main purpose or objective of the study must be made explicit in this section.

Materials and methods: a detailed description of the procedure, with materials, location, dates, statistics, etc. This description must be sufficiently detailed so that other researchers may replicate the study. If a novel procedure is used, it must be thoroughly explained.

Results: presents major findings in an organized and appropriate manner. Avoids the use of excessively long tables.

Discussion: most relevant, troublesome or novel points of the study are highlighted, and major results are explained in relation to the importance of the study and contributions to its field.

Conclusions: final reflections about the study, with a clear relation to its purpose and objectives, frequently pointing towards future actions and research.

Acknowledgements: Straightfoward and short paragraph between text of manuscript and Literature cited. Mention funding or support of the project. Avoid titles such as Dr., Lic., etc.

Literature cited: Follow APA citation norms (American Psychological Association Publications Manual, Sixth Edition). List of references must only include those that are cited within the text. Order the references in alphabetical order, and chronologically in the case of a sole author. If there are many references of a same author (s) in the same year, add letters a, b, c, etc. to the year. Do not abbreviate names of journals. Include all authors. This section must be at the end of the manuscript. 


\section{Citation examples}

\section{Article in journals:}

Antonelli, A., Nylander, J. A., Persson, C. \& Sanmartín, I. (2009). Tracing the impact of the Andean uplift on Neotropical plant evolution. Proceedings of the National Academy of Sciences, 106(24): 9749-9754.

\section{Books:}

Gutiérrez, F. P. (2010). Los recursos hidrobiológicos $y$ pesqueros en Colombia. Bogotá: Instituto de Investigación de Recursos Biológicos Alexander von Humboldt. 118 pp.

\section{Thesis:}

Cipamocha, C. A. (2002). Caracterización de especies y evaluación trófica de la subienda de peces en el raudal Chorro de Córdoba, bajo río Caquetá, Amazonas, Colombia. (Thesis). Bogotá D. C.: Universidad Nacional de Colombia, Facultad de Ciencias, Departamento de Biología.

\section{Technical reports:}

Andrade, G. I. (2010). Gestión del conocimiento para la gestión de la biodiversidad: bases conceptuales y propuesta programática para la reingeniería del Instituto Humboldt. (Technical report). Instituto de Investigación de Recursos Biológicos Alexander von Humboldt. Bogotá D. C., 80 pp.

\section{Book or report chapter:}

Fernández F., Palacio, E. E. \& MacKay, W. P. (1996). Introducción al estudio de las hormigas (Hymenoptera: Formicidae) de Colombia. In Amat, G. D., Andrade, G. \& Fernández, F. (Eds.). Insectos de Colombia. Estudios Escogidos. Pp: 349-412. Bogotá: Academia Colombiana de Ciencias Exactas, Físicas y Naturales \& Centro Editorial Javeriano.

\section{Congress, symposium or workshop summary:}

Señaris, J. C. (2001). Distribución geográfica y utilización del hábitat de las ranas de cristal (Anura; Centrolenidae) en Venezuela. Presented in Programa y Libro de Resúmenes del IV Congreso Venezolano de Ecología, Mérida, Venezuela. p. 124.

\section{Law or decree:}

Congreso de Colombia. (February 8th 1994) Ley General de Educación. [Ley 115 de 1994]. DO: 41.214.

\section{Web pages:}

Must be clearly included in the text of the manuscript, but not be included in Literature cited section.

\section{Details for Data Papers}

A Data Paper is a type of scientific publication that was designed to stimulate the publication of biodiversity data. Data Papers give academic and professional acknowledgement to those who intervene, in one way or another, in the management of information about biodiversity, as well as highlight the existence and importance of data sets to the rest of the scientific community.

As its name suggests, a Data Paper describes a primary data set. Although a Data Paper is not, strictly speaking, a scientific investigation, it must contain relevant information about the data set (objectives, methods for data collection, funding, taxonomic and geographic coverage, etc.), along with its value and utility (basic or applied) for the scientific community (Chavan \& Penev, 2011) ${ }^{1}$. The great advantage and novelty of this type of manuscript is that it is linked to the data set through a stable and trustworthy repository, the IPT (Integrated Publishing Toolkit). Also, the data set is supported by metadata also available through the IPT and linked to the Data Paper.

A Data Paper must be submitted only when the linked data are primary and original data that have a temporal and methodological restriction and are available in data aggregators such as $\underline{\mathrm{SiB} \text { Colombia }}$ and GBIF. Data must be able to follow the Darwin Core $(\mathrm{DwC})$ standard. Examples of such data sets include:

- Project observations

- Biological collections

- Species lists

\footnotetext{
${ }^{1}$ Chavan, V. y Penev, L. (2011). The data paper: The mechanism to incentivize data publishing in biodiversity science. BMC Bioinformatics 2011, 12(Sup. 15): S2
} 
- Genomic data

- Samples

- Inventories

- Databases

- Functional traits

Data sets that do not comply with the characteristics mentioned above will not be accepted for publication as a Data Paper. Such is the case of compilations of biological records that come from secondary sources (p. e. from published literature).

\section{Preparation of Data Paper (publication of data and creation of manuscript)}

Since the purpose of a Data Paper is to describe all available data resources regarding biodiversity, it must always be linked to the data set it describes through an URL or DOI.

Information about how to generate and submit a manuscript in order for it to be considered as a Data Paper by using the tools and publication model of SiB Colombia is found below. It must be noted, however, that Biota Colombiana also accepts Data Papers that link to data sets published in other known platforms as long as it is linked to a trustworthy repository and has an IPT link. The parts of a Data Paper manuscript are described in Table 1.
As other types of manuscripts that are submitted to the journal, Data Papers will be reviewed by peers and must comply with the same format specifications, citation norms and use of language. Similarly, Data Papers must also be presented with a cover letter, as mentioned in the present Guidelines for authors. Have in mind that as soon as the manuscript is submitted and under evaluation, described data must be available in a public online repository with an adequate license of use and attribution.

\section{Step 1}

\section{Data publication in SiB Colombia}

$\mathrm{SiB}$ Colombia uses a publication model pased on the IPT as its working tool. Using the IPT, the first version of the manuscript may be generated in rich text format (RTF), based on its associated metadata. This tool is available as long as the data set has been indexed by $\mathrm{SiB}$ Colombia and sufficient metadata are linked (more information on publication process of $\mathrm{SiB}$ Colombia may be consulted in https://www.sibcolombia.net/).

A. Registration of organisation. To publish through SiB Colombia, your organisation must be registered as a publishing partner. Consult this link to find already registered organisations. If your organisation is not registered, adding an organisation is easy through the Registration Format.

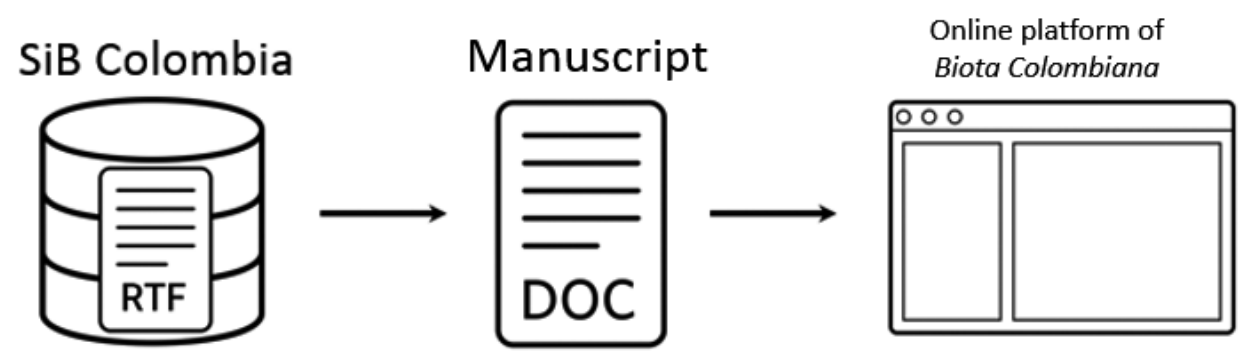

Figura 1. General process to submit a Data Paper from SiB Colombia to the journal Biota Colombiana. 
B. Data standardization. Data must be structured in a table using the Darwin Core $(\mathrm{DwC})$ standard. Download respective template that is appropriate for type of data or generate your template.

C. Data quality. Data quality must be verified and improved using available tools to identify and correct possible geographical, taxonomic or format errors, among others.

D. Online upload of data. IPT is a tool that shares different types of biodiversity data as long as data is structured using DwC standard. To upload data to the IPT, you must have an existing user account in the available IPT of SiB Colombia. If you do not have an account, you may contact the SiB Colombia Coordinating Team (EC-SiB) and request an account to the email address sib@humboldt.org.co.

E. Data mapping. Once the data set is uploaded, verify that it follows DwC elements. For more information, consult the IPT User Manual or contact EC-SiB.

F. Creation of metadata. Metadata structure is similar to that of a traditional research article. In this way, metadata has the same general structure of a Data Paper and thus facilitate the generation of the manuscript. In the metadata section of the IPT, all information that broadens the context of data must be included. There is a total of 12 sections to add information as metadata to describe the data set. For more information, consult the IPT User Manual or contact EC-SiB.

G. Publish resource and notify EC-SiB. Once all previous instructions are completed, IPT will be activate the "Publish" option. Click on the button and send an e-mail to sib@humboldt.org.co in order to notify EC-SiB about your publication. The e-mail must have as subject "Published resource" and include:

- Name

- Name of organisation

- Name of published resource
- URL of general view of resource after publication

Now data are indexed by SiB Colombia and GBIF, and have a digital object identifier, DOI.

Step 2

Creation of manuscript for submission as Data Paper through IPT

The IPT used for publishing the data set generates a RTF manuscript that describes the data set. The link to the data set in the manuscript appears under the title "Data published through GBIF". Here you will find step by step information about how to generate a manuscript based on the data set metadata published in SiB Colombia.

- On the resource homepage published in IPT, click on the RTF button to download the first version of the manuscript in rich text format, which may be opened in any text processor (p. e. Word) (Figure 2).

- Downloaded manuscript is in English. Necessary corrections to follow Biota Colombiana guidelines must be completed on the template. Data Papers template may be downloaded here.

- Once the manuscript is adjusted with additional text, tables and figures, it may be submitted to the journal Biota Colombiana through its online platform, following steps of registry as a user. The complete editorial process is developed through this platform.

\section{Step 3}

Adjustments and corrections of manuscript for data paper

When a manuscript is submitted as a Data Paper, it will go through the same peer review process as other articles of the journal, with specifications for Data Paper evaluation.

After evaluation, and if the manuscript is accepted, it will be returned to the author with the comments of reviewers and the journal's Editor so that respective modifications may be incorporated. 


\section{Escarabajos coprófagos de la cuenca alta y media del río Bita, Vichada (Colombia)}

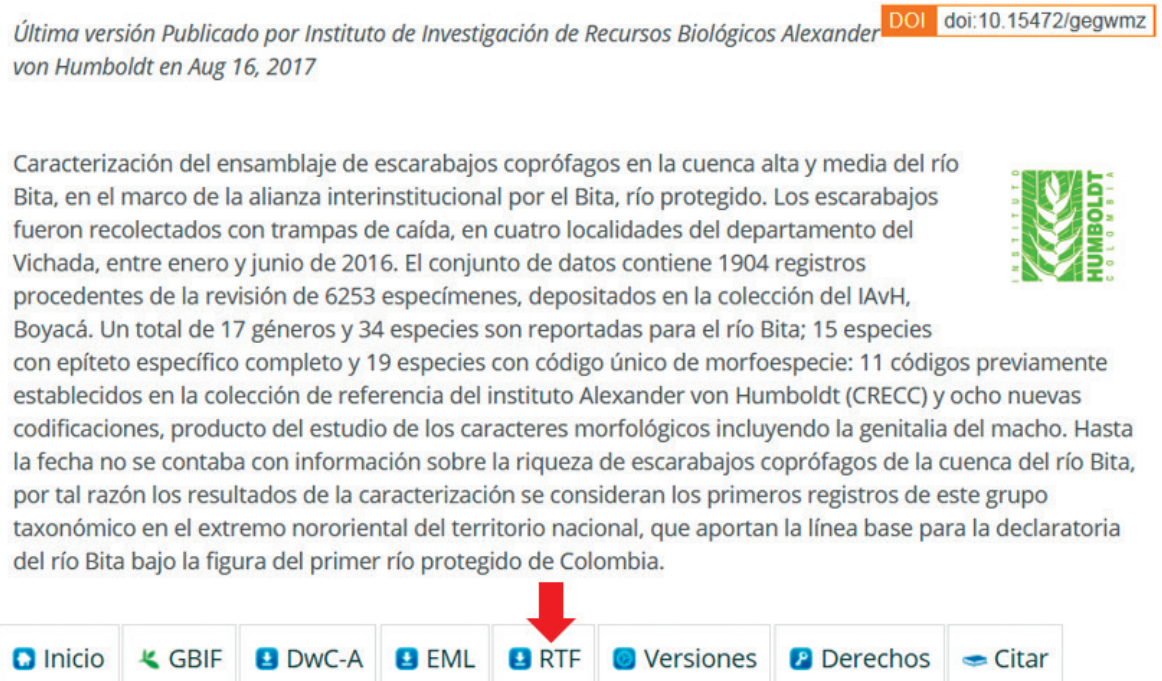

Figure 2. Metadata of a data set may be downloaded from the IPT as a RTF file, giving the first version of the manuscript that will be submitted to the journal.

As the author, you should do all corrections or modifications directly on the IPT metadata and not on the manuscript. In this way, the metadata of the linked data set are also improved by the peer review and editorial comments.

Once metadata in the IPT are improved, the resource publication must be updated so the changes are reflected. On the resource homepage of the published resource, click on the RTF button to download the improved version of the manuscript in rich text format that may be opened in any type of text processor (p. e. Word).
After the manual changes of additional text, figures and tables, and the corroboration that the manuscript follows all of the journal's requirements, it must be sent again through the online platform of Biota Colombiana.

\section{Parts of a Data Paper}

Data Papers differ from other articles that are published in the journal Biota Colombiana in the sections it should include and are mentioned in Table 1. 
Table 1. Structure of a Data Paper and correspondence with GMP elements of IPT.

Name of section

\section{Title}

Authors

Affiliations

\section{Correspondence with IPT elements}

Derived from the element Title. Centered and without period at the end.

Derived from the elements Resource creators, Metadata providers and Associated Parties. From the elements, the combination of name and last name, separated by a coma, is created. Author affiliations are indicated with numbers $(1,2,3 \ldots)$ at the end of each last name with a superscript. Centered.

Derived from the elements Resource creators, Metadata providers and Associated Parties. From these elements, the combination of organisation, address, postal code, city, country and email address constitute the complete affiliation. If one or more authors share the same affiliation, it is represented with the same number.

Derived from the elements Resource creators and Metadata providers. From the elements, the combination of name, last name, and email address is created. Email addresses are inside parentheses. If there is more than one author as contact, authors are separated by comas. If the Resource creator and Metadata provider is the same author, the Resource creator is assumed to be the contact. Text is centered.

\section{Dates of received, revised, accepted and published}

Manually incorporated by the editorial assistant of the journal to indicate respective dates of when the manuscript was received, revised, accepted and published as a Data Paper in Biota Colombiana.

\begin{tabular}{|c|c|}
\hline Abstract & $\begin{array}{l}\text { Derived from the element description. Abstract must be included in Spanish or } \\
\text { Portuguese, and English. }\end{array}$ \\
\hline Keywords & $\begin{array}{l}\text { Derived from the element keywords. Words are separated by comas. Keywords must be } \\
\text { written in Spanish or Portuguese, and English. }\end{array}$ \\
\hline Introduction & Not derived and must be added by the authors manually. \\
\hline Taxonomic coverage & $\begin{array}{l}\text { Derived from section of taxonomic coverage: description, scientific names, common names } \\
\text { and category. }\end{array}$ \\
\hline Geographic coverage & $\begin{array}{l}\text { Derived from section of geographic coverage: description, minimum latitude, maximum } \\
\text { latitude, minimum longitude, maximum longitude. }\end{array}$ \\
\hline Temporal coverage & Derived from section of temporal coverage: description, start date, end date. \\
\hline Project description & $\begin{array}{l}\text { Derived from section of project data: title, project personnel, funding, study area description, } \\
\text { design description. }\end{array}$ \\
\hline Collection data & $\begin{array}{l}\text { Derived from section of collection data: name of collection, collection identifier, parental } \\
\text { collection identifier, specimen preservation methods, curatorial units. }\end{array}$ \\
\hline Materials and methods & $\begin{array}{l}\text { Derived from section of sampling methods: study extent, study description, quality control, } \\
\text { step description. }\end{array}$ \\
\hline Results & -- \\
\hline Data description & $\begin{array}{l}\text { Derived from external links, among others: name, file URL, file format, version of file format, } \\
\text { publication date, language, copyright. An additional description of data such as text, figures } \\
\text { and tables may be added. }\end{array}$ \\
\hline Additional information & Derived from element of additional information. \\
\hline Discussion & Not derived and must be added by the authors manually. \\
\hline Acknowledgements & Not derived and must be added by the authors manually. \\
\hline Literature cited & Derived from element of citations. \\
\hline
\end{tabular}




\section{Biota Colombiana}

Volumen 19 (Sup. 1) - Colombia Bio y otras novedades científicas

Una publicación del / A publication of: Instituto de Investigación de Recursos Biológicos Alexander von Humboldt

En asocio con / In collaboration with:

Instituto de Ciencias Naturales de la Universidad Nacional de Colombia

Instituto de Investigaciones Marinas y Costeras - Invemar

Missouri Botanical Garden

\section{TABLA DE CONTENIDO/ TABLE OF CONTENTS}

Editorial

Una nueva especie de barniz de pasto Elaeagia (Rubiaceae), de la cordillera Oriental de Colombia. A new species of Elaeagia (Rubiaceae) from the cordillera Oriental of Colombia. Humberto Mendoza-Cifuentes y José Aguilar-Cano

Una nueva especie de Allomaieta (Melastomataceae - Cyphostyleae) del piedemonte amazónico de los Andes de Colombia. A new species of Allomaieta (Melastomataceae-Cyphostyleae) from the Amazonian foothills of the Colombian Andes. Humberto Mendoza-Cifuentes

Dos nuevas especies de Miconia (Melastomataceae) del piedemonte oriental de la cordillera Central de Antioquia, Colombia. Two new species of Miconia (Melastomataceae) from the eastern foothills of the Cordillera Central of Antioquia, Colombia. Humberto Mendoza-Cifuentes, Julián Aguirre-Santoro y Álvaro Idárraga

Dos nuevas especies de árboles molinillo (Magnolia: Magnoliaceae) de la serranía de los Yariguíes, departamento de Santander, Colombia. Two new species of "molinillo" tree (Magnolia: Magnoliaceae) from Serranía de los Yariguíes, Santander, Colombia. José Aguilar-Cano, Humberto Mendoza-Cifuentes y Melisa Ayala-Joya

Catálogo de la flora de los Parques Nacionales de Colombia: Parque Nacional Natural El Tuparro. Catalogue of the flora of the National Natural Parks of Colombia: El Tuparro National Natural Park. Humberto Mendoza-Cifuentes y Mireya P. Córdoba-Sánchez .

Primer registro del efemeróptero Oligoneuria (Oligoneurioides) amazonica (Demoulin, 1955) (Insecta: Ephemeroptera, Oligoneuriidae) para Colombia. First record of Mayfly Oligoneuria (Oligoneurioides) amazonica (Demoulin, 1955) (Insecta: Ephemeroptera, Oligoneuriidae) from Colombia. Cristian E. Granados-Martínez, Carlos A. Lasso y Juan M. Fuentes-Reinés .....

Variaciones morfológicas y algunas notas bioecológicas del cangrejo de agua dulce Neostrengeria charalensis Campos y Rodríguez, 1985 (Decapoda: Pseudothelphusidae), en ambientes exo y endocársticos de los Andes colombianos. Morphological variations and some bioecological notes of the freshwater crab Neostrengeria charalensis Campos \& Rodríguez, 1985 (Decapoda: Pseudothelphusidae), in exo and endocárstic environments of the Colombian Andes. Martha R. Campos, Ada Acevedo, Carlos A. Lasso y Jesús Fernández-Auderset

Ectoparásitos (Argulidae, Cymothoidae, Corallanidae) en rayas de agua dulce (Potamotrygonidae) de la Orinoquia colombiana. Ectoparasites (Argulidae, Cymothoidae, Corallanidae) in freshwater rays (Potamotrygonidae) of the Colombian Orinoquia. Carlos A. Lasso, Martha R. Campos, Mónica A. Morales-Betancourt y David Castro .....

Trichomycterus rosablanca (Siluriformes, Trichomycteridae) a new species of hipogean catfish from the Colombian Andes. Trichomycterus rosablanca (Siluriformes, Trichomycteridae) una especie nueva de bagre hipogeo de los Andes colombianos. Lina M. Mesa S., Carlos A. Lasso, Luz E. Ochoa y Carlos DoNascimiento

A new species of cave catfish, genus Trichomycterus (Siluriformes: Trichomycteridae), from the Magdalena River system, Cordillera Oriental, Colombia. Una nueva especie de bagre de caverna, género Trichomycterus (Siluriformes: Trichomycteridae), del sistema río Magdalena, cordillera Oriental, Colombia. César A. Castellanos-Morales

Una nueva rana de huesos verdes del género Scinax (Anura: Hylidae) asociada a los bosques subandinos de la cuenca del río Magdalena, Colombia. A new frog with green bones of the genus Scinax (Anura: Hylidae), associated with the sub-Andean forests of the Magdalena River basin, Colombia. Andrés R. Acosta-Galvis

Una nueva rana nodriza (Anura: Dendrobatidae) de los bosques de niebla asociados a la cuenca del Orinoco de Colombia. A new nurse frog (Anura: Dendrobatidae) from the cloud forests of the Orinoco basin of Colombia. Andrés R. Acosta-Galvis y Adrián Pinzón

\section{Notas}

Nuevos registros de plantas acuáticas para la región Guayana y notas sobre las islas flotantes en el río Guaviare, Guainía, Colombia. New records of aquatic plants from the Guayana region in Colombia, with notes on floating islands in the Guaviare River, Guainía. Anabel Rial

Primer registro del hemíptero Strudivelia cinctipes Champion, 1898 (Hemiptera: Veliidae) para ambientes cavernícolas de Colombia. First record of the hemiptera Strudivelia cinctipes Champion, 1898 (Hemiptera: Veliidae) for cave environments in Colombia. Hernán Aristizábal-García, Natalia Herreño-Castellanos y Carlos A. Lasso 